\title{
funSentiment at SemEval-2017 Task 4: Topic-Based Message Senti- ment Classification by Exploiting Word Embeddings, Text Features and Target Contexts
}

\author{
Quanzhi Li, Armineh Nourbakhsh, Xiaomo Liu, Rui Fang, Sameena Shah \\ Research and Development \\ Thomson Reuters \\ 3 Times Square, NYC, NY 10036 \\ \{quanzhi.li, armineh.nourbakhsh, xiaomo.liu, rui.fang, sameena.shah\}@ thomsonreuters.com
}

\begin{abstract}
This paper describes the approach we used for SemEval-2017 Task 4: Sentiment Analysis in Twitter. Topic-based (target-dependent) sentiment analysis has become attractive and been used in some applications recently, but it is still a challenging research task. In our approach, we take the left and right context of a target into consideration when generating polarity classification features. We use two types of word embeddings in our classifiers: the general word embeddings learned from 200 million tweets, and sentiment-specific word embeddings learned from 10 million tweets using distance supervision. We also incorporate a text feature model in our algorithm. This model produces features based on text negation, tf.idf weighting scheme, and a Rocchio text classification method. We participated in four subtasks (B, C, D \& $\mathrm{E}$ for English), all of which are about topic-based message polarity classification. Our team is ranked \#6 in subtask B, \#3 by $\mathrm{MAE}^{\mathrm{u}}$ and \#9 by $\mathrm{MAE}^{\mathrm{m}}$ in subtask C, \#3 using RAE and \#6 using KLD in subtask $\mathrm{D}$, and \#3 in subtask E.
\end{abstract}

\section{Introduction}

There have been many studies on message or sentence level sentiment classification (Go et al., 2009; Mohammand et al., 2013; Pang et al., 2002; Liu, 2012; Tang et al., 2014), but there are few studies on target-dependent, or topic-based, sentiment prediction (Jiang et al., 2011; Dong et al., 2014; Vo and Zhang, 2015). A target entity in a message does not necessarily have the same polarity type as the message, and different entities in the same message may have different polarities. For example, in the tweet "Linux is better than Windows", the two named entities, Linux and Windows, will have different sentiment polarities. In this paper, we describe our approach for the subtask B, C, D \& E of
SemEval-17 Task 4: Sentiment Analysis in Twitter (Sara Rosenthal and Noura Farra and Preslav Nakov, 2017). All the four subtasks are on topic-based message sentiment classification. Task $\mathrm{B}$ and $\mathrm{C}$ are about topic-based message polarity classification. Given a message and a topic, in task B, we classify the message on a two-point scale: positive or negative sentiment towards the topic. And in task C, we classify the message on a five-point scale: sentiment conveyed by the tweet towards the topic on a five-point scale. Task D and E are about Tweet quantification. Given a set of tweets about a given topic, in task $\mathrm{D}$, we want to estimate the distribution of the tweets across two-point scale - the positive and negative classes, and in task $\mathrm{E}$, we estimate that on a five-point scale - the five classes of a fivepoint scale. Our approach uses word embeddings (WE) learned from general tweets, sentiment specific word embeddings (SSWE) learned from distance supervised tweets, and a weighted text feature model (WTM).

Learning features directly from tweet text has recently gained lot of attention. One approach is to generate sentence representations from word embeddings. Several word embedding generation algorithms have been proposed in previous studies (Collobert et al., 2011; Mikolov et al., 2013). Using the general word embeddings directly in sentiment classification is not effective, since they mainly model a word's semantic context, ignoring the sentiment clues in text. Therefore, words with opposite polarity, such as worst and best, are mapped onto vectors embeddings that are close to each other in some dimensions. Tang et al. (2014) propose a sentiment-specific word embedding (SSWE) method for sentiment analysis, by extending the word embedding algorithm. 
SSWE encodes sentiment information in the word embeddings.

In our approach, we incorporate WE, SSWE and a weighted text feature model (WTM) together. The WTM model generates two types of features. The first type is a negation feature based on the negation words in a tweet. The second set of features is created by computing the similarity between the tweet and each of the polarity types, using cosine similarity and the tf.idf word weighting scheme. Each polarity category is represented by a pseudo centroid tweet learned from training data. This is very similar to the Rocchio text classifier (Christopher et al., 2008), but here all the similarity values with all the polarity types are used as features, and fed to the classification algorithm. The rationale behind the second set of features is that the similarity values with the different polarity types will have some correlations, and using all of them as features will provide more information to the classifier. For example, a positive tweet usually will have a higher similarity value with neutral type than with the negative type. This will provide an additional signal to the classifier.

The context of an entity will affect its polarity value, and usually an entity has a left context and also a right one, unless it is at the beginning or end of a message. Both the context information and the interaction between these two contexts are included in the classification features of our approach. Our approach uses both SSWE and WE to represent these contexts, since WE and SSWE complement each other, and our experiment shows that using both increases the accuracy by more than $6 \%$, compared to using only one of them.

In the following sections, we present the related studies, our methodology and the experiments and results for subtask B, C, D and E.

\section{Related Work}

Message Level Sentiment: Traditional sentiment classification approaches use sentiment lexicons (Mohammad et al., 2013; Thelwall et al., 2012; Turney, 2002) to generate various features. Pang et al. treat sentiment classification as a special case of text categorization, by applying learning algorithms (2002). Many studies follow Pang's approach by designing features and applying different learning algorithms on them (Feldman, 2013; Liu, 2012). Go et al. (2009) proposed a distance supervision approach to derive features from tweets obtained by positive and negative emotions. Some studies (Hu et al., 2013; Liu, 2012; Pak and Paroubek 2010) follow this approach. Feature engineering plays an important role in tweet sentiment classification; Mohammad et al. (2013) implemented hundreds of hand-crafted features for tweet sentiment classification.

Deep learning has been used in the sentiment analysis tasks, mainly by applying word embeddings (Collobert et al., 2011; Mikolov et al., 2013). Learning the compositionality of phrase and sentence and then using them in sentiment classification is also explored by some studies (Hermann and Blunsom, 2013; Socher et al., 2011; Socher et al., 2013). Using the general word embeddings directly in sentiment classification may not be effective, since they mainly model a word's semantic context, ignoring the sentiment clues in text. Tang et al. (2014) propose a sentiment-specific word embedding method by extending the word embedding algorithm from (Collobert et al., 2011) and incorporating sentiment data in the learning of word embeddings.

Target-dependent Sentiment: Jiang et al. (2011) use both entity dependent and independent features generated based on a set of rules to assign polarity to entities. By using POS features and the CRF algorithm, Mitchell et al. (2013) identify polarities for people and organizations in tweets. Dong et al. (2014) apply adaptive recursive neural network on the entity level sentiment classification. These two approaches use syntax parsers to parse the tweet to generate related features. In our approach, we consider both the left and right contexts of a target when generating features.

\section{Methodology}

In this section, we describe the three main components used in our method, the WE, SSWE and WTM models, and how they are integrated together.

\subsection{Word Embedding}

Word embedding is a dense, low-dimensional and real-valued vector for a word. The embeddings of a word capture both the syntactic structure and semantics of the word. Traditional bag-of-words and bag-of-n-grams hardly capture the semantics of words. Word embeddings have been used in many NLP tasks. The C\&W model (Collobert et al., 2011) and the word2vec model (Mikolov et al., 2013), which is used in this 
study to generate the WE embeddings, are the two popular word embedding models.

The embeddings are learned to optimize an objective function defined on the original text, such as likelihood for word occurrences. One implementation is the word2vec from Mikolov et al. (2013). This model has two training options, continuous bag of words and the Skip-gram model. The Skip-gram model is an efficient method for learning high-quality distributed vector representations that capture a large number of precise syntactic and semantic word relationships. This model is used in our method and here we briefly introduce it.

The training objective of the Skip-gram mod$\mathrm{el}$ is to find word representations that are useful for predicting the surrounding words in a sentence or a document. Given a sequence of training words $W_{1}, W_{2}, W_{3}, \ldots, W_{N}$, the Skip-gram model aims to maximize the average log probability

$$
\frac{1}{N} \sum_{n=1}^{N} \sum_{-m \leq i \leq m, i \neq 0} \log p\left(W_{n+i} \mid W_{n}\right)
$$

where $m$ is the size of the training context. A larger $m$ will result in more semantic information and can lead to a higher accuracy, at the expense of the training time. Generating word embeddings from text corpus is an unsupervised process. To get high quality embedding vectors, a large amount of training data is necessary. After training, each word, including all hashtags in the case of tweet text, is represented by a lowdimensional, dense and real-valued vector.

\subsection{Sentiment-Specific Word Embedding}

The C\&W model (Collobert et al., 2011) learns word embeddings based on the syntactic contexts of words. It replaces the center word with a random word and derives a corrupted n-gram. The training objective is that the original $n$-gram is expected to obtain a higher language model score than the corrupted n-gram. The original and corrupted n-grams are treated as inputs of a feedforward neural network, respectively.

SSWE extends the C\&W model by incorporating the sentiment information into the neural network to learn the embeddings; it captures the sentiment information of sentences as well as the syntactic contexts of words (Tang et al., 2014). Given an original (or corrupted) n-gram and the sentiment polarity of a tweet as input, it predicts a two-dimensional vector $\left(f_{0}, f_{1}\right)$, for each input n-gram, where $\left(f_{0}, f_{1}\right)$ are the language model score and sentiment score of the input n-gram, respectively. The training objectives are twofold: the original $n$-gram should get a higher language model score than the corrupted n-gram, and the polarity score of the original n-gram should be more aligned to the polarity label of the tweet than the corrupted one. The loss function is the linear combination of two losses - $\operatorname{loss}_{0}\left(t, t^{\prime}\right)$ is the syntactic loss and $\operatorname{loss}_{1}\left(t, t^{\prime}\right)$ is the sentiment loss:

$$
\operatorname{loss}\left(t, t^{\prime}\right)=\alpha * \operatorname{loss}_{0}\left(t, t^{\prime}\right)+(1-\alpha) * \operatorname{loss}_{1}\left(t, t^{\prime}\right)
$$

The SSWE model used in this study was trained from massive distant-supervised tweets, collected using positive and negative emotions.

\subsection{Weighted Text Feature Model (WTM)}

WTM features: This model only uses the training data set to generate features; it does not use any external lexicon or other data sources, such as embeddings learned from millions of tweets. The feature generation process is simple, fast, and effective. This model generates two types of features for each training or test tweet:

- Negation feature - the number of negation words in the tweet. This is different from other studies that add a prefix to all the words that follow the negation word, e.g. NOT xxx becomes NOT_xxx. We use the following negation words: no, not, cannot, rarely, seldom, neither, hardly, nor, n't, never

- Features corresponding to the cosine similarity values between this tweet and the pseudo centroid tweet of each of the polarity types.

Pseudo centroid tweet: The pseudo centroid tweet for each sentiment type is built from training data, via the following steps:

- Tweet text is pre-processed as follow:

$\circ$ all URLs and mentions are removed

o dates are converted to a symbol

o all ratios are replaced by a special symbol

$\circ$ integers and decimals are normalized to two special symbols

- all special characters, except hashtags, emoticons, question marks and exclamations, are removed

o negation words that are already used in the negation features are removed

- A tf.idf value is calculated for each term in a polarity category. For tf.idf, each catego- 
ry is treated as a document, and tf is normalized by its category size.

- A pseudo centroid tweet is generated for each sentiment type. We define a centroid as a vector containing the tf.idf value for each term in this category. Although we call it a "tweet", its length is much longer than a regular tweet.

Similarity value: For each training or test tweet, its similarity with a sentiment type is calculated as follows:

- The tweet text is pre-processed using the same steps mentioned above

- A tf.idf value is calculated for each remaining term

- A cosine similarity is calculated between this tweet and each sentiment type.

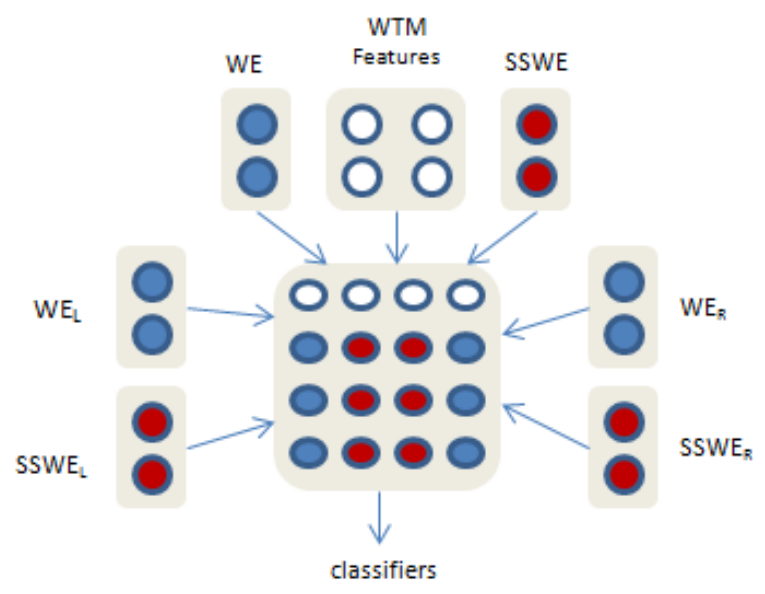

Figure 1. The features generated from different models.

\subsection{Feature Generation}

\subsubsection{Features}

Given a tweet and the target entity, eight types of features are generated based on WE, SSWE, and WTM models. They are integrated together to train the classifier. Figure 1 shows the eight types of features. Six types of features are generated from WE and SSWE embeddings for a target entity. Two types of features are generated from the WTM model. The red ones are SSWE embeddings, and the blue ones are WE embeddings. The subscript letter ${ }_{\mathrm{L}}$ and ${ }_{\mathrm{R}}$ refer to the left and right side of an entity, respectively. They are described below:

$\mathbf{W E}_{\mathbf{L}}$ and $\mathbf{W E}_{\mathbf{R}}$ : These are the $\mathrm{WE}$ embeddings for the text on the left side and right side of the target entity, respectively. In the four subtasks, occasionally, the given topic (target) is a paraphrase of the actual target entity in the tweet text, and it is not easy to match these two. In this case, the whole tweet text is used for both the left and right contexts, and this case is handled in the same way when generating $\mathrm{SSWE}_{\mathrm{L}}$ and $\mathrm{SSWE}_{\mathrm{R}}$ described below.

SSWE $_{\mathbf{L}}$ and SSWE $_{\mathbf{R}}$ : These are the SSWE embeddings for the text on the left side and right side of the target entity, respectively.

WE and SSWE: these are the embeddings generated from the whole message text, which means they are entity independent features. We use these two features to capture the whole message, which reflects the interaction between the left and right sides of the entity.

WTM features: It has two types of features: the negation feature and the features corresponding to the cosine similarity values between the tweet and the pseudo centroid tweet of each of the polarity types. We have described how to generate them in the previous section.

These eight types of features together capture different types of information we are interested: the entity's left and right contexts, the interaction of the two sides, the sentiment specific word embedding information, the general word embedding information, and the sentiment affected by negation terms.

\subsubsection{Text Representation from Term Embeddings}

There are different ways to obtain the representation of a text segment, such as a whole tweet or the left/right context of an entity, from word embeddings. In our approach, we use the concatenation convolution layer, which concatenates the layers of max, min and average of word embeddings, because this layer gives the best performance based in our pilot experiments.

\begin{tabular}{|c|c|c|c|}
\hline Subtask & \multicolumn{3}{|c|}{ Metric, Score \& Rank } \\
\hline \multirow{2}{*}{ B } & $\boldsymbol{\rho}$ & $\boldsymbol{F}_{\boldsymbol{I}}{ }^{\boldsymbol{N}}$ & $\boldsymbol{A c c}$ \\
\cline { 2 - 4 } & $0.834_{6}$ & $0.824_{8}$ & $0.827_{8}$ \\
\hline \multirow{2}{*}{ C } & $\boldsymbol{M A E}^{\boldsymbol{\mu}}$ & \multicolumn{2}{|c|}{$\boldsymbol{M A E}^{\boldsymbol{m}}$} \\
\cline { 2 - 4 } & $0.530_{3}$ & $0.0 .842_{9}$ \\
\hline \multirow{2}{*}{ D } & $\boldsymbol{R A E}$ & $\boldsymbol{A E}$ & $\boldsymbol{K L D}$ \\
\cline { 2 - 4 } & $0.939_{3}$ & $0.109_{6}$ & $0.060_{6}$ \\
\hline \multirow{2}{*}{ E } & \multicolumn{3}{|c|}{$\boldsymbol{E M D}$} \\
\cline { 2 - 4 } & \multicolumn{3}{|c}{$0.273_{3}$} \\
\hline
\end{tabular}

Table 1. Evaluation result for subtask B, C, D \& E. The subscript of each score is the rank of our approach by the corresponding metric for that task. 


\section{Experiments and Results}

\subsection{WE Model Construction}

The tweets for building the WE model include tweets obtained through Twitter's public streaming API and the Decahose data (10\% of Twitter's streaming data) obtained from Twitter. Only English tweets are included in this study. Totally there are about 200 million tweets. Each tweet text is preprocessed to get a clean version, following similar steps described in the WTM model subsection, except the stop removal step. Stop words are not removed, since they provide important information on how other words are used. Totally, about 2.9 billion words were used to train the WE model. Based on our pilot experiments, we set the embedding dimension size, word frequency threshold and window size as 300, 5 and 8, respectively. There are about 1.9 million unique words in this model.

\subsection{SSWE Model Construction}

The SSWE model for Twitter was trained from massive distant-supervised tweets (Tang et al., 2014), collected using positive and negative emoticons, such as :), =), :( and :-(. A total of 10 million tweets were collected, where 5 million contain positive emotions and the other 5 million contain negative ones. The embedding dimension size was set as 50 and the window size as 3 .

\subsection{Data Set and Results for Task 4}

For subtask $\mathrm{B}$ and $\mathrm{D}$, the sentiment classification and quantification based on a 2-point scale, the training data is from the related tasks of SemEval-2015 and SEmEval-2016. There are 20,538 tweets, but the actual tweet texts are not provided, due to privacy concerns. So we crawled these tweets from Twitter's REST API. However, we were unable to obtain all these tweets because some of them were already deleted or not available due to authorization status change. To build our classifier, we split this data set into three parts: $70 \%$ as training data, $20 \%$ as development data and $10 \%$ for testing our classifier. For these two subtasks, we applied several classification algorithms, such as SMO, LibLinear and logistic regression, to see which one performs the best. The result we reported is based on logistic regression, which performed the best.

For subtask $\mathrm{C}$ and $\mathrm{E}$, the sentiment classification and quantification based on a 5-point scale, the training data is from the related tasks of SemEval-2016. There are 30,632 tweets, and similarly to subtask B and D, we downloaded the tweets from Twitter's API and split them into three parts. The result we reported is based on SMO (Keerthi et al., 2001), which performed the best among several classifiers we tested. SMO is a sequential minimal optimization algorithm for training a support vector classifier.

Table 1 shows the results of our approach for the four subtasks. It lists the scores and ranks of our team for all the performance metrics used for each subtask. The subscript of each score is the rank of our team using that metric for that subtask.

The meanings of the measures used in Table 1 are explained below:

For task $\boldsymbol{B}: \boldsymbol{\rho}$ is the macro-averaged recall, which is macro-averaged over the positive and the negative class. Accuracy and $\mathrm{F} 1$ measures are also used for subtask B. As subtask B is topicbased, each metric is computed individually for each topic, and then the results are averaged across the topics to yield the final score. This is the same for all the measures used in task C, D and $\mathrm{E}$, which are all topic based tasks.

For task $C: \mathrm{MAE}^{\mathrm{M}}$ is the macro-averaged mean absolute error, which is an ordinal classification measure. Note that $\mathrm{MAE}^{\mathrm{M}}$ is a measure of error, not accuracy, and thus lower values are better. $M A E^{\mu}$ is an extension of macro-averaged recall for ordinal regression. More details about these two measures are described in (Baccianella et al., 2009; Nakov et al., 2016a).

For task D: KLD is the Kullback-Leibler Divergence measure, a measure of error, which means that lower values are better. $\mathrm{AE}$ is the absolute error and RAE is the relative absolute error.

For task E: Subtask E is an ordinal quantification task. As in binary quantification, the goal is to compute the distribution across classes, assuming a quantification setup. EMD is Earth Mover's Distance (Rubner et al., 2000), which is currently the only known measure for ordinal quantification. Like KLD and MAE, EMD is a measure of error, so lower values are better.

\section{Conclusion}

This paper describes the approach we used for subtask B, C, D and E of SemEval-2017 Task 4: Sentiment Analysis in Twitter. We use two types of word embeddings in our classifiers: general word embeddings learned from 200 million tweets, and sentiment-specific word embeddings learned from 10 million tweets using distance supervision. We also incorporate a 
weighted text feature model in our algorithm. Our team is ranked \#6 in subtask B, \#3 using $\mathrm{MAE}^{\mathrm{u}}$ metric and \#9 using $\mathrm{MAE}^{\mathrm{m}}$ metric in subtask C, \#3 using RAE and \#6 using KLD in subtask D, and \#3 in subtask E.

\section{References}

Stefano Baccianella, Andrea Esuli, Fabrizio Sebastiani, SentiWordNet 3.0: An Enhanced Lexical Resource for Sentiment Analysis and Opinion Mining, LREC 2010

Stefano Baccianella, Andrea Esuli, and Fabrizio Sebastiani. 2009. Evaluation measures for ordinal regression. In Proceedings of the 9th IEEE International Conference on Intelligent Systems Design and Applications (ISDA 2009). Pisa, IT, pages 283-287.

Ronan Collobert. Jason Weston. Leon Bottou. Michael Karlen. Korav Kavukcuoglu. and Pavel Kuksa. Natural language nrocessing (almost) from scratch. Journal Machine Learning Research, 12:2493-2537, 2011.

L. Dong, F. Wei, C. Tan, D. Tang, M. Zhou, K. Xu, adaptive recurisive neural network for targetdepedant twitter sentiment classification. In Proceedings of ACL 2014.

R.-E. Fan, K.-W. Chang, C.-J. Hsieh, X.-R. Wang, and C.-J. Lin. LIBLINEAR: A Library for Large Linear Classification, Journal of Machine Learning Research, 9(2008), 1871-1874.

Ronen Feldman. 2013. Techniques and applications for sentiment analysis. Communications of the ACM, 56(4):82-89.

Alec Go, Lei Huang, and Richa Bhayani. 2009. Twitter sentiment analysis. Final Projects from CS224N for Spring 2008/2009 at The Stanford Natural Language Processing Group

Karl M. Hermann and Phil Blunsom. 2013. The role of syntax in vector space models of compositional semantics. In Proceedings ACL, pages 894-904

Xia Hu, Jiliang Tang, Huiji Gao, and Huan Liu. 2013. Unsupervised sentiment analysis with emotional signals. In Proceedings of the International World Wide Web Conference, pages 607-618.

L. Jiang, M. Yu, M. Zhou, X. Liu, T. Zhao, targetdepedant twitter sentiment classification, In Proceedings of ACL 2011

S.S. Keerthi, S.K. Shevade, C. Bhattacharyya, K.R.K. Murthy, 2001. Improvements to Platt's SMO Algorithm for SVM Classifier Design. Neural Computation. 13(3):637-649.

Bing Liu. 2012. Sentiment analysis and opinion mining. Synthesis Lectures on Human Language Technologies, 5(1):1-167

Tomas Mikolov, Ilya Sutskever, Kai Chen, Greg Corrado, and Jeffrey Dean. Distributed Representations of Words and Phrases and their Compositionality. In Proceedings of NIPS, 2013.

Christonher Manning. Prabhakar Raghavan and Hinrich Schütze, (2008), Vector space classification. Introduction to Information Retrieval. Cambridge University Press.

Margaret Mitchell, Jacqui Aguilar,Theresa Wilson, and Benjamin Van Durme. Open domain targeted sentiment. I Proceedings of EMNLP, 2013.

Saif M Mohammad, Svetlana Kiritchenko, and Xiaodan Zhu. 2013. Nrc-canada: Building the state-ofthe- art in sentiment analysis of tweets. In Proceedings of SemEval 2013.

P. Nakov, A. Ritter, S. Rosenthal, F. Sebastiani and V. Stoyanov. 2016. SemEval-2016 Tasks: Sentiment Analysis in Twitter. In Proceedings of SemEval 2016

Alexander Pak and Patrick Paroubek. 2010. Twitter as a corpus for sentiment analysis and opinion mining. In Proceedings of Language Resources and Evaluation Conference, volume 2010.

Bo Pang, Lillian Lee, and Shivakumar Vaithyanathan. 2002. Thumbs up? sentiment classification using machine learning techniques. In Proceedings of EMNLP, pages 79-86

J. Platt, Fast Training of Support Vector Machines using Sequential Minimal Optimization. In B. Schoelkopf and C. Burges and A. Smola, editors, Advances in Kernel Methods - Support Vector Learning, 1998

Sara Rosenthal and Noura Farra and Preslav Nakov, SemEval-2017 Task 4: Sentiment Analysis in Twitter. In Proceedings of SemEval 2017, Vancouver, Canada

Yossi Rubner, Carlo Tomasi, and Leonidas J. Guibas. 2000. The Earth Mover's Distance as a metric for image retrieval. International Journal of Computer Vision, 40(2):99-121.

Richard Socher, J. Pennington, E.H. Huang, A.Y. Ng, and C.D. Manning. 2011. Semi-supervised recursive autoencoders for predicting sentiment distributions. In Proceedings of EMNLP 2011, pages $151-161$.

Richard Socher, Alex Perelygin, Jean Wu, Jason Chuang, Christopher D. Manning, Andrew Ng, and Christopher Potts. 2013. Recursive deep models for semantic compositionality over a sentiment treebank. In Proceedings of EMNLP 2013, pages $1631-1642$.

Duyu Tang, Furu Wei, Nan Yang, Ming Zhou, Ting Liu, and Bing Qin. 2014. Learning sentimentspecific word embedding for twitter sentiment classification. In Proceedings of $A C L$ 2014, pages 1555-1565.

Duyu Tang, Bing Qin, Xiaocheng Feng, Ting Liu, 2016. Effective LSTMs for Target-Dependent Sentiment Classification, In Proceedings of COLING 2016

Mike Thelwall, Kevan Buckley, and Georgios Paltoglou. 2012. Sentiment strength detection for the social web. Journal of the American Society for Information Science and Technology, 63(1):163-173.

Peter D Turney. 2002. Thumbs up or thumbs down?: semantic orientation applied to unsupervised classification of reviews. In Proceedings of $A C L$, pages $417-424$

D. Vo and Y. Zhang, Target-dependant twitter sentiment classification with rich automatic features, IJCAI 2015.

Sida Wang and Christopher D Manning. 2012. Baselines and bigrams: Simple, good sentiment and topic classification. In Proceedings of $A C L$ 2012, pages 90-94 